\title{
Long-term efficacy of oral alendronate therapy in an elderly patient with polyostotic fibrous dysplasia: A case report
}

\author{
GUI-DONG LI, AKIRA OGOSE, TETSUO HOTTA, HIROYUKI KAWASHIMA, \\ TAKASHI ARIIZUMI, YONGJUN XU and NAOTO ENDO \\ Division of Orthopedic Surgery, Graduate School of Medical and Dental Sciences, \\ Niigata University, Niigata 951-8510, Japan
}

Received April 1, 2011; Accepted July 19, 2011

DOI: $10.3892 / 01.2011 .369$

\begin{abstract}
Polyostotic fibrous dysplasia (PFD) is a highturnover bone disease that frequently entails chronic bone pain, pathological fractures and severe deformities. Recently, bisphosphonates have shown effective antiresorptive properties in the treatment of children or adults with PFD. We report on a 79-year-old female with PFD, who had severe lower limb deformity and chronic bone pain in multiple sites of her extremities for more than 55 years. The patient experienced significant decrease in bone pain and bone turnover markers following long-term (8.5 years) treatment with a low-dose oral alendronate treatment $(5 \mathrm{mg} / \mathrm{day})$. To the best of our knowledge, this is the first report of a long-term follow-up of a postmenopausal elderly patient with long-standing symptomatic PFD following continuous low-dose oral alendronate therapy. This case report indicates that long-term daily administration of low-dose alendronate alone is a potential treatment option for elderly patients with PFD, particularly those with long-standing bone pain.
\end{abstract}

\section{Introduction}

Fibrous dysplasia (FD) of bone is an osteoblastic lineage disease that is caused by a post-zygotic activating mutation in the gene that encodes the $\alpha$-subunit of the stimulatory G-protein (1). The mutated osteoblasts lead to excessive osteoclastic activity and increased bone resorption in FD, similar to that observed in high-turnover bone diseases including Paget's disease and osteoporosis, and this pathological hallmark provides a rationale for the use of bisphosphonates, such as dronate and alendronate, potent antiresorptive drugs, in patients with FD (2). Numerous studies have noted that intravenous pamidronate therapy alone or in combination with

Correspondence to: Dr Akira Ogose, Division of Orthopedic Surgery, Graduate School of Medical and Dental Sciences, Niigata University, 1-757 Asahimachi-dori, Niigata, Niigata 951-8510, Japan E-mail: aogose@med.niigata-u.ac.jp

Key words: fibrous dysplasia of bone, bisphosphonate, alendronate, bone turnover oral alendronate markedly relieved bone pain, improved the radiological aspects, increased bone density and decreased bone turnover in children or adults with polyostotic fibrous dysplasia (PFD) (3-6). A number of studies have documented that oral alendronate alone had similar positive results in patients with PFD or McCune-Albright syndrome (MAS) (3,7-10). In this case report, we describe a 79-year-old postmenopausal female with PFD, who had severe lower limb deformities and had experienced multiple sites of chronic bone pain for over 55 years. This patient demonstrated a significant improvement in pain relief and a reduction in bone turnover markers following 102 months of daily oral alendronate treatment alone.

\section{Case report}

A female patient had developed a limp in her right leg at 12 years of age (1943). At the age of 16 (1947), moderate bone pain had started in her right lower leg, and subsequently, chronic bone pain developed in her bilateral hip, thigh and left lower leg. At 27 years of age (1958), she first presented to Niigata University due to persistent severe bone pain and a distinct deformity of her right hip (Fig. 1A). She was diagnosed as having PFD and received a subtrochanteric osteotomy of her right femur. At the age of 33 (1964), the patient sustained a pathological fracture of her right proximal femur and was successfully treated by cast immobilization. At the age of 37 (1969), she experienced another fracture in the left femoral neck, and this fracture was treated by nail plate fixation with a fibular bone graft. The pathological examination of biopsy tissues showed the characteristic pattern of FD. Mild to moderate (sometimes severe) bone pain in multiple sites lasted for over 33 years.

In 2001 , at the age of 70 , the patient developed dysbasia and severe bone pain again in her left hip that lasted more than 4 months due to the subcutaneous prominence of the nail and an incomplete cortical fracture. The original plate was replaced by an AO condyle dynamic screw plate. Tissue from the intraoperative biopsy showed an activating mutation of the GNAS1 (Gs $\alpha$ ) gene, and there was no maturation in the non-lamellar woven bone or in the fibrous matrix (Fig. 1B). Following the last surgery the patient was able to walk outside with a T-cane, although the multiple sites of 

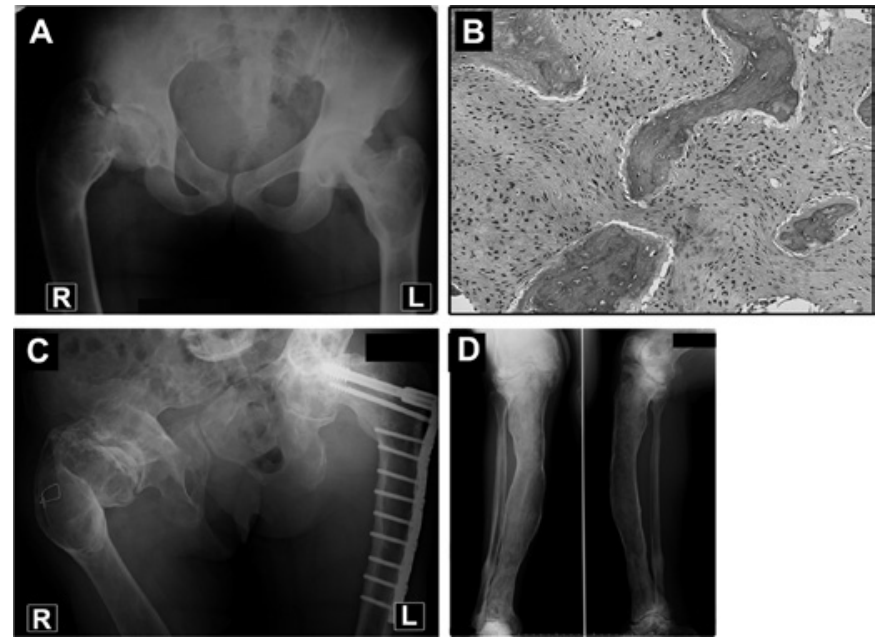

Figure 1. Radiological and histological findings. (A) An anteroposterior radiograph of the pelvis and proximal femur in a female patient with polyo-stotic fibrous dysplasia taken at the patient's first presentation (at the age of 27). There is a classic shepherd's crook deformity of the right femur with cortical thinning and a typical ground-glass appearance in the bilateral femur. (B) The histological appearance of the bone lesion of the right femoral neck (at the age of 70) shows typical fibroblastic stroma and immature woven bones without rimming of osteoblasts (hematoxylin-eosin; $\mathrm{x} 100)$. (C and D) Radiographs taken at 102 months after starting oral alendronate therapy (at the age of 79). (C) An anteroposterior view of the pelvis and proximal femur, and (D) an anteroposterior (left panel) and lateral (right panel) view of the right tibia revealed no significant cortical thickening or bone formation.

bone pain continued to interfere with her daily activities. Since 2002 , at 71 years of age, she has been receiving oral alendronate sodium at $5 \mathrm{mg} /$ day $102 \mathrm{months}$ as of the writing of this manuscript). The changes in bone pain were evaluated using the visual pain analog scale (0-10) at every visit. Biochemical measurements, including serum total alkaline phosphatase (ALP), calcium and phosphate, were performed at approximately 2-month intervals. Serum bone alkaline phosphatase (BAP) and urine $\mathrm{N}$-terminal cross-linked telopeptide of type I collagen (NTX) were also examined 79-102 months following initiation of therapy. Radiographs of the involved bone lesions were taken at one-year intervals. Changes in the lumber spine (L2-L4) bone mineral density (BMD) were measured using dual-energy X-ray absorptiometry (DEXA).

On physical examination prior to alendronate treatment, there were moderate restrictions of the patient's range of motion in both sides of her hip joints, but she had no reduction in muscle force of the extremities. She had tenderness in her bilateral proximal femora and right tibia. The pain levels were moderate in the bilateral hips and right lower leg, and were mild in the left knee and the right ankle (Table I).

Radiological examinations at pretreatment indicated that the deformity of the right femur and the radiolucent lesions in multiple sites had progressed compared to her first visit. Fibrous dysplastic lesions were also detected in her skull, right first rib, right humerus, right ulna, right hand, pubic bones, left tibia and right foot by $99 \mathrm{mTc}-\mathrm{methylene}$ diphosphonate (MDP) bone scan. The lumber spine BMD value was $0.900 \mathrm{~g} / \mathrm{cm}^{2}$ (T-score, -0.4 ) at pretreatment (Table II).

The patient's bone turnover markers prior to treatment showed high levels of serum ALP, 602 IU/l (reference
Table I. Changes in pain levels after 102 months of oral alendronate treatment.

\begin{tabular}{lcc}
\hline & \multicolumn{2}{c}{ Visual analog pain scale } \\
\cline { 2 - 3 } Sites & Before treatment & Posttreatment \\
\hline Left hip & 6 & 1 \\
Right hip & 5 & 1 \\
Left knee & 3 & 0 \\
Right lower leg & 5 & 1 \\
Right ankle & 3 & 0 \\
\hline
\end{tabular}

Table II. Changes in the lumbar spine (L2-L4) BMD after 89 months of oral alendronate treatment.

\begin{tabular}{lcccc}
\hline & $\begin{array}{c}\text { Age } \\
\text { (years })\end{array}$ & $\begin{array}{c}\text { BMD } \\
\left(\mathrm{g} / \mathrm{cm}^{2}\right)\end{array}$ & $\begin{array}{c}\text { Mean } \\
\text { T-score }\end{array}$ & $\begin{array}{c}\text { BMD } \\
\text { changes vs. } \\
\text { baseline }\end{array}$ \\
\hline $\begin{array}{l}\text { Pretreatment } \\
\begin{array}{l}\text { Posttreatment } \\
(6 \text { months })\end{array}\end{array}$ & 71 & 0.900 & -0.4 & \\
$\begin{array}{l}\text { Posttreatment } \\
(89 \text { months })\end{array}$ & 78 & 1.135 & 1.1 & $26.2 \%$ \\
\hline
\end{tabular}

BMD, bone mineral density.

range $125-259$ by the JSCC method); BAP, $88.3 \mathrm{U} / 1$ (reference range 9.6-35.4); and urine NTX, $281.7 \mathrm{nmol} \mathrm{BCE} / \mathrm{mmol} \mathrm{Cr}$ (reference range 8.3-69.9 nmol BCE/mmol Cr). The levels of serum calcium and serum phosphate at pretreatment were slightly lower than the normal range, $8.4 \mathrm{mg} / \mathrm{dl}$ (normal range $8.7-10.0 \mathrm{mg} / \mathrm{dl}$ ) and $2.4 \mathrm{mg} / \mathrm{dl}$ (normal range $2.5-4.6 \mathrm{mg} / \mathrm{dl}$ ), respectively. No other abnormalities were found during the biochemical analysis.

Following oral alendronate treatment, the bone pain in her multiple sites was markedly reduced during the first 8 months, and was then completely relieved or has remained at a mild level up to the most recent follow-up (Table I). The high pretreatment level of serum ALP decreased by 24 months after treatment initiation and then decreased gradually to a near normal level (275 IU/l at the final examination) (Fig. 2A). The high pretreatment level of serum BAP was decreased to within the normal range after 102 months of initiating the treatment ( $35 \mathrm{U} / 1$ at the final examination) (Fig. 2B). The urine NTX also markedly decreased from a high pretreatment level to a lower one at 102 months posttreatment $(102.5 \mathrm{nmol} \mathrm{BCE} / \mathrm{mmol} \mathrm{Cr}$ at the final examination) (Fig. 2C). The serum calcium level was slightly lower than the normal level during the first 17 months of treatment, but was then maintained consistently at a normal level (Fig. 3A). The serum phosphate level oscillated between normal and slightly low levels between 17 and 55 months after treatment was initiated, but remained stable within the normal range following treatment (Fig. 3B). 
$\mathbf{A}$

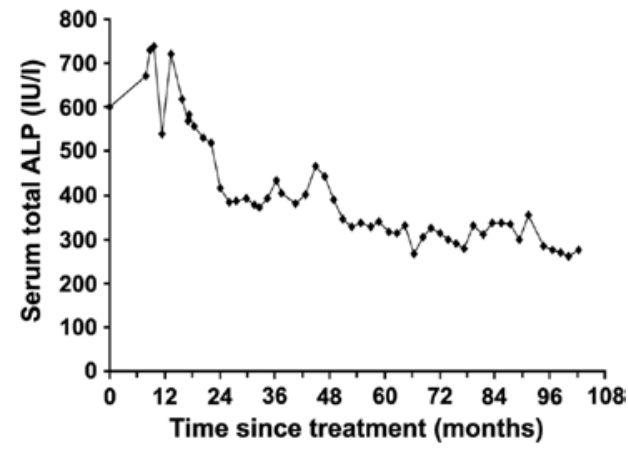

B

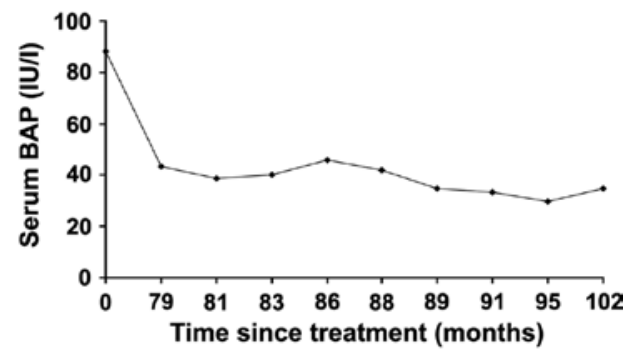

C

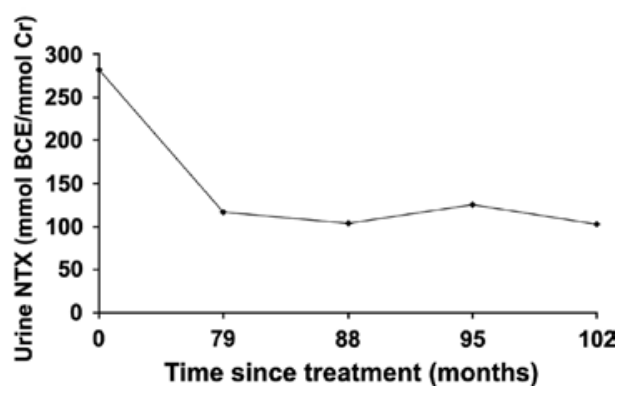

Figure 2. Changes in bone turnover markers during the 102 months of oral alendronate treatment. (A) The high pretreatment level of serum total alkaline phosphatase (ALP) decreased rapidly during the 13-24 month period following alendronate treatment, followed by a slower and continued decrease up to 102 months after initiation of treatment. (B) The high level of pretreatment serum bone alkaline phosphatase (BAP) was markedly decreased and remained persistently within the normal range at 89-102 months posttreatment. (C) The urine N-terminal cross-linked telopeptide of type I collagen (NTX) level was decreased to significantly below the pretreatment level during the course of alendronate treatment. Data for the serum BAP and the urine NTX after 0-79 months of treatment were not available.

Following 89 months of alendronate treatment, the value of BMD had increased by $26.2 \%$ (mean BMD, $1.135 \mathrm{~g} / \mathrm{cm}^{2}$; T-score, 1.1) when compared with the pretreatment value (Table II). Although there was no clear evidence of cortical thickening or refilling of the fibrocystic bone lesions during the 102 months of alendronate treatment (Fig. 1C and D), the patient has not suffered from any pathological fractures during the treatment, and is capable of walking outside with a T-cane for several hours without resting. No adverse drug reactions occurred following oral alendronate administration, and the medication is currently being continued.

\section{Discussion}

Few case reports exist on the long-term follow-up of elderly patients with long-standing symptomatic PFD (11-13), and there are also few studies on the ambulation of elderly PFD patients with severe lower extremity deformities $(11,13)$. Although some of these patients had fairly severe deformities,
$\mathbf{A}$

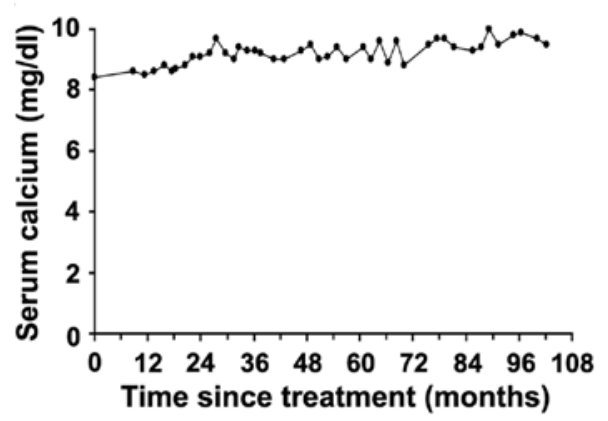

B

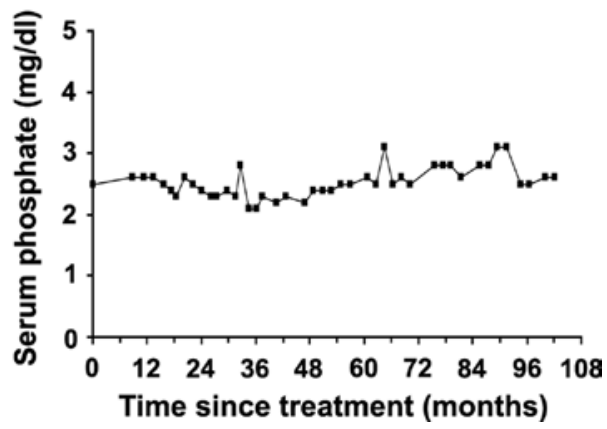

Figure 3. Changes in the serum levels of calcium and phosphate during the 102 months of oral alendronate treatment. (A) The value of serum calcium was slightly lower than the normal range during the first 17 months of the treatment, but was then maintained consistently within the normal range. (B) The value of serum phosphate fluctuated between the normal level and slightly lower levels during the period between 17 and 55 months after the initiation of treatment, and stabilized within the normal range by 102 months posttreatment.

they were functionally active and were able to walk with aids $(11,13)$. In the present case, total follow-up has continued for over 52 years since the first diagnosis of PFD and, in spite of the severe shepherd's crook deformity in her later years, the patient was able to walk outside using a cane for a considerable amount of time. However, multiple sites of chronic bone pain had lasted for over 55 years until she received oral bisphosphonate therapy.

Nitrogen-containing bisphosphonates such as pamidronate and alendronate are drugs that prevent osteoclast-mediated bone resorption, and are widely used to treat osteoporosis and similar diseases, such as Paget's disease of the bone. Numerous open clinical studies with high-dose intravenous infusions of pamidronate have shown that they may yield favorable results in patients with symptomatic FD (3-6). Successful outcomes have also been reported for the use of oral alendronate without combination with intravenous infusion in a few patients with PFD (3,7-10). These patients were treated with different doses of alendronate: $70 \mathrm{mg}$ weekly in a 10.5-year-old female (10); $20 \mathrm{mg}$ daily in a 22 -year-old male (7); $10 \mathrm{mg}$ daily in 34- and 39-year-old females (3); $5 \mathrm{mg}$ daily in a 3-year-old female (8) and a 45-year-old premenopausal female (9). These regimens led to significant improvements in pain relief and substantial reductions in the levels of bone turnover markers, with the exception of 2 adult patients who did not show any changes in urine NTX $(3,9)$. A distinct increase in BMD by bone scanning was also noted $(7,8)$. However, improvements in cortical thickening and filling of the bone lesions were not apparent $(3,9)$. In the 
present study, the patient was treated with low-dose $(5 \mathrm{mg}$ / day) oral alendronate alone for more than 102 months (8.5 years) since the age of 71 , and this treatment period was far longer than that of previous studies, in which a maximum period of 2 years was recorded. To the best of our knowledge, this is the first report of the use of long-term oral alendronate treatment in a postmenopausal elderly patient with PFD. The patient's long-standing bone pain was almost completely relieved during the period beginning 8 months after starting oral alendronate therapy.

The levels of total serum ALP and serum BAP are frequently elevated in patients with PFD and MAS, and in certain cases, these bone formation markers are significantly higher than in normal subjects $(4,11)$. In the present case, the pretreatment levels of serum ALP and BAP were also relatively high, but the levels markedly declined to near normal and normal ranges, respectively, during oral alendronate treatment. The urinary NTX, which is known to be one of the most sensitive predictors of bone resorption in bisphosphonate therapy, also decreased significantly following alendronate treatment. Along with the decrease in the levels of bone turnover markers, the mean BMD value at the lumbar spine was increased markedly by $26.2 \%$. However, the BMD at the involved lesions was not available in this study. By contrast, no clear effects were noted on cortical thickening and filling of lytic lesions in the radiographic aspects. This negative radiological finding following long-term alendronate treatment in our case was similar to the findings described in previous studies, indicating that the intravenous administration of pamidronate did not result in any clear improvement in the radiological appearance (14) and had no effect on the skeletal burden (15). However, our patient has not suffered from any pathological fractures during the alendronate treatment period.

In conclusion, the data from the current case suggest that long-term daily administration of low-dose alendronate alone is capable of providing satisfactory results with regard to reduction of bone pain and bone turnover, and may increase the BMD in patients with PFD. Although it appears likely that oral alendronate alone cannot cure FD itself, this regimen is a preferred option for the treatment of patients exhibiting PFD symptoms, particularly those patients with long-standing bone pain.

\section{References}

1. Marie PJ, de Pollak C, Chanson P and Lomri A: Increased proliferation of osteoblastic cells expressing the activating Gs alpha mutation in monostotic and polyostotic fibrous dysplasia. Am J Pathol 150: 1059-1069, 1997.

2. Riminucci M, Kuznetsov SA, Cherman N, Corsi A, Bianco P and Gehron Robey P: Osteoclastogenesis in fibrous dysplasia of bone: In situ and in vitro analysis of IL-6 expression. Bone 33: 434-442, 2003.

3. Lane JM, Khan SN, O'Connor WJ, et al: Bisphosphonate therapy in fibrous dysplasia. Clin Orthop 382: 6-12, 2001.

4. Isaia GC, Lala R, Defilippi C, et al: Bone turnover in children and adolescents with McCune-Albright syndrome treated with pamidronate for bone fibrous dysplasia. Calcif Tissue Int 71: 121-128, 2002.

5. Chapurlat RD, Hugueny P, Delmas PD and Meunier PJ: Treatment of fibrous dysplasia of bone with intravenous pamidronate: long-term effectiveness and evaluation of predictors of response to treatment. Bone 35: 235-242, 2004

6. Chapurlat RD: Medical therapy in adults with fibrous dysplasia of bone. J Bone Miner Res 21 (Suppl 2): 114-119, 2006.

7. Yamomoto T, Ozono K, Shima M, Yoshikawa H and Okada S: Alendronate and pharmacological doses of 1 alpha OHD3 therapy in a patient with McCune-Albright syndrome and accompanying hypophosphatemia. J Bone Miner Metab 20: 170-173, 2002.

8. Khadilkar VV, Khadilkar AV and Maskati GB: Oral bisphosphonates in polyostotic fibrous dysplasia. Indian Pediatr 40: 894-896, 2003.

9. Kitagawa $\mathrm{Y}$, Tamai $\mathrm{K}$ and Ito $\mathrm{H}$ : Oral alendronate treatment for polyostotic fibrous dysplasia: a case report. J Orthop Sci 9: 521-525, 2004.

10. Aragão AL and Silva IN: Oral Alendronate Treatment for Severe Polyostotic Fibrous Dysplasia due to McCune-Albright Syndrome in a Child: A Case Report. Int J Pediatr Endocrinol: Oct 4, 2010 (E-pub ahead of print).

11. Harris WH, Dudley HR Jr and Barry RJ: The natural history of fibrous dysplasia. An orthopaedic, pathological, and roentgenographic study. J Bone Joint Surg Am 44: A207-A233, 1962.

12. Sissons HA and Malcolm AJ: Fibrous dysplasia of bone: case report with autopsy study 80 years after the original clinical recognition of the bone lesions. Skeletal Radiol 26: 177-183, 1997.

13. Szendrói M, Rahóty P, Antal I and Kiss J: Fibrous dysplasia associated with intramuscular myxoma (Mazabraud's syndrome): a long-term follow-up of three cases. J Cancer Res Clin Oncol 124: 401-406, 1998.

14. Plotkin H, Rauch F, Zeitlin L, Munns C, Travers R and Glorieux FH: Effect of pamidronate treatment in children with polyostotic fibrous dysplasia of bone. J Clin Endocrinol Metab 88: 4569-4575, 2003.

15. Collins MT, Kushner H, Reynolds JC, et al: An instrument to measure skeletal burden and predict functional outcome in fibrous dysplasia of bone. J Bone Miner Res 20: 219-226, 2005. 